\title{
Network Collaborative Modeling Design of Intelligent Products in Industrial Design System CNC Machine Tools
}

\author{
Fan Li \\ Architecture College, Changjiang Vocational College, Wuhan, 430074, China \\ fan_li2013@yeah.net
}

Keywords: Artistic design, Fuzzy control, Artificial intelligence, Joint simulation, MATLAB software, Solidworks

\begin{abstract}
In the design process of product art modeling image, this paper uses the fuzzy cognitive theory of output variables to do artificial intelligence operations on the products art design, which achieves a virtual intelligent simulation. The decoupling process of artificial intelligence uses MATLAB to code, and establishes a data connection using MATLAB toolbox and Solidworks, which realizes the visualization operation in the design process. Through the analysis of the system testing and design case, artificial intelligent algorithms can effectively realize cognitive thinking storage, and play a feedback effect in product shape artistic image optimization design, which provides a technical reference for the study on product art design.
\end{abstract}

\section{Introduction}

With the progress of times and the development of science and technology, people's aesthetic ideas have improved and changed, and the product appearance design moves to high level of development and changes constantly. Many factors influence the product design; however, modern product modeling design emphasis to meet the needs of people and society, which makes beautiful appearance and exquisite pleasant product to service the people's lives and production activities, and improves the material and spiritual civilization of the whole society [1,2]. The use of artificial intelligence can not only realize the cognitive learning, but also realize the optimization design of product image. The main process is as follows:

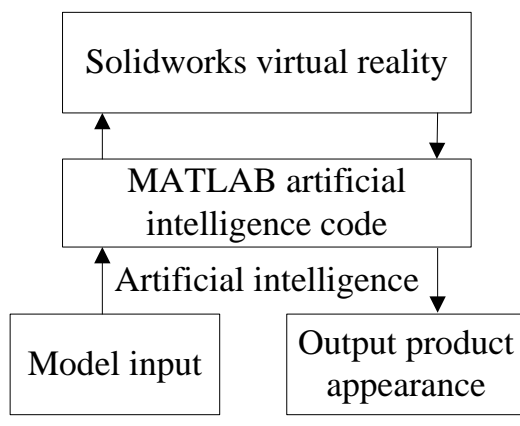

Fig.1: The design process of product image artificial intelligent

The design process of product image artificial intelligence uses software virtual simulation technology, which makes product design with qualitative and quantitative analysis virtual operation environment [3]. Through MATLAB data interface, input the command and output the appearance image, so as to realize the product appearance design. Through the control of MATLAB artificial intelligence code, system can store the learning and memory trace, forming the standard reference system, finally realizes the art image optimization design using feedback regulation function.

\section{Product Modeling Cognitive Theory based on Artificial Intelligence Algorithms}

In order to make the artificial intelligence of product art modeling design more generally applicable, this paper designs a kind of PI online independent adjustment parameter model based on cognitive thinking, through the feedback regulation, the artificial intelligence design is more optimization [46]. The discrete expressions of intelligent decoupling are as follows: 


$$
Z(x)=y_{p}(x) \times e(x)+y_{i}(x) \times \sum_{n=0}^{x} e(n)
$$

The corresponding correction parameter is:

$$
\left\{\begin{array}{c}
y_{p}(x)=y_{p}(x-1)+\Delta y_{p}(x) \\
y_{i}(x)=y_{i}(x-1)+\Delta y_{i}(x)
\end{array} .\right.
$$

Where $y_{p}(x)$ and $y_{i}(x)$ are the intelligent output of proportional parameters and integral parameter in intelligent operation step $x ; y_{p}(x-1)$ and $y_{i}(x-1)$ are the intelligent output of proportional parameters and integral parameter in intelligent operation step; $\Delta X_{p}(x)$ and $\Delta X_{i}(x)$ are the feedback amount of artificial intelligence control, and the two dimension continuous function is:

$$
\left\{\begin{array}{l}
y_{p}(x)=f_{1}(e, e k) \\
y_{i}(x)=f_{2}(e, e k)
\end{array} .\right.
$$

The output variables fuzzy domain $\Delta y_{p}(x)$ and $\Delta y_{i}(x)$ can be defined as shown in the formula (4):

$$
\Delta X_{p}(x), \Delta X_{i}(x)=\{-n,-n-1 \ldots . . n, n+1\} .
$$

Using the fuzzy cognitive of output variables to do artificial intelligence operation on the product artistic design, and artificial intelligent decoupling control process can be realized by using MATLAB artificial intelligence programming code, and establishing data results with Solidworks [7]. The specific procedures are as follows:

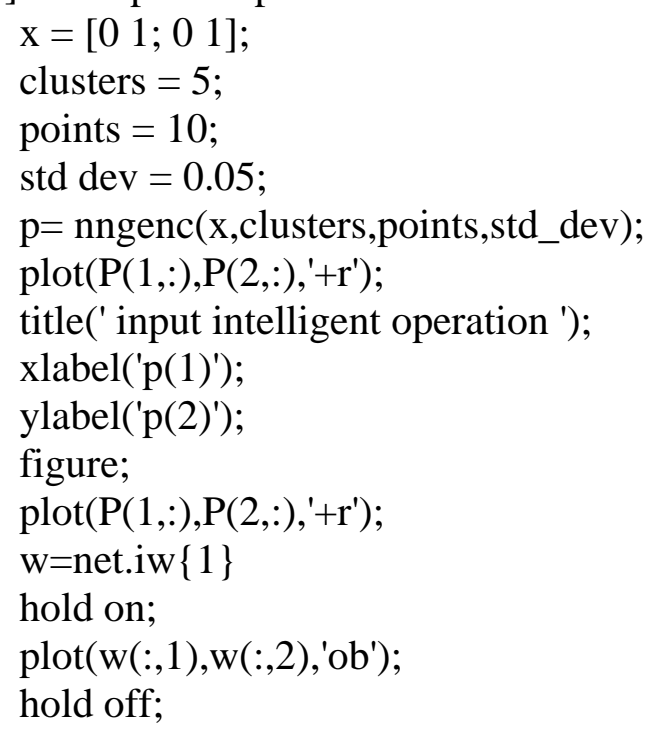

\section{The Design of Product Image Art Modeling Artificial Intelligent}

In order to verify effectiveness and reliability of artificial intelligent algorithm in section second, this paper uses the MATLAB and Solidworks CO simulation to do simulation design on the product image art form, and the process is as shown in Figure 2. 


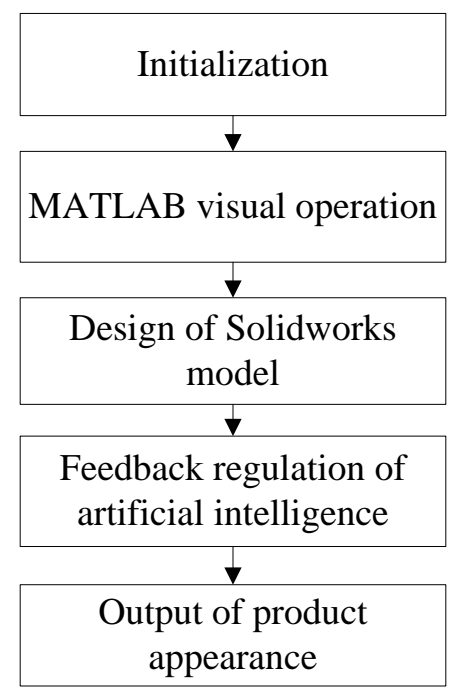

Fig.2: The design process of product appearance artificial intelligent

Arbitrarily selected 46 samples and tested, the output value of the network through the approximation can achieve expected value, of which 3 samples corresponding to the input of the network, the desired output and the actual output as shown in Table 1.

Table 1: Simulation of neural network

\begin{tabular}{llllllllll}
\hline $\mathrm{T}$ & $\mathrm{T} 2$ & $\mathrm{~T} 3$ & $\mathrm{~T} 4$ & $\mathrm{~L}$ & $\mathrm{~L}$ & $\mathrm{~L}$ & $\mathrm{~S} 1$ & $\mathrm{~S} 2$ & $\mathrm{~S} 3$ \\
1 & & & & 1 & 2 & 3 & & & \\
\hline 0. & 0. & 0. & 0. & 0 & 0 & 1 & 0.0422 & 0.0306 & 0.9673 \\
1 & 3 & 2 & 1 & & & & & & \\
0. & 0. & 0. & 0. & 0 & 1 & 0 & 0.0139 & 0.9563 & 0.0383 \\
2 & 3 & 4 & 2 & & & & & & \\
0 & 0. & 0 & 0. & 1 & 0 & 0 & 0.9840 & 0.0027 & 0.0252 \\
& 3 & & 4 & & & & & & \\
$\mathrm{~T}$ & $\mathrm{~T} 2$ & $\mathrm{~T} 3$ & $\mathrm{~T} 4$ & $\mathrm{~L}$ & $\mathrm{~L}$ & $\mathrm{~L}$ & $\mathrm{~S} 1$ & $\mathrm{~S} 2$ & $\mathrm{~S} 3$ \\
1 & & & & 1 & 2 & 3 & & & \\
0. & 0. & 0. & 0. & 0 & 0 & 1 & 0.0422 & 0.0306 & 0.9673 \\
1 & 3 & 2 & 1 & & & & & & \\
0. & 0. & 0. & 0. & 0 & 1 & 0 & 0.0139 & 0.9563 & 0.0383 \\
2 & 3 & 4 & 2 & & & & & & \\
0 & 0. & 0 & 0. & 1 & 0 & 0 & 0.9840 & 0.0027 & 0.0252 \\
& 3 & & 4 & & & & & & \\
\hline
\end{tabular}

Among them, T1-T4 is the road characteristic value; L1-L3 is network expected output; S1-S3 is the actual output network.

By the data obtained and Figure 3, Figure 4 and Figure 5 we can see, through the trained network processing, actual output are close to the expected output. By the approximation we can obtain expected output from the actual output, it means according to actual output of the network we can judge intelligent vehicles' type, the correct rate of network training is $100 \%$. The trained network can be used as intelligent vehicle path identification tool[8,9]. At the same time, BP network construction is simple, practical, which can be trained and tested at any time according to actual condition of road. 


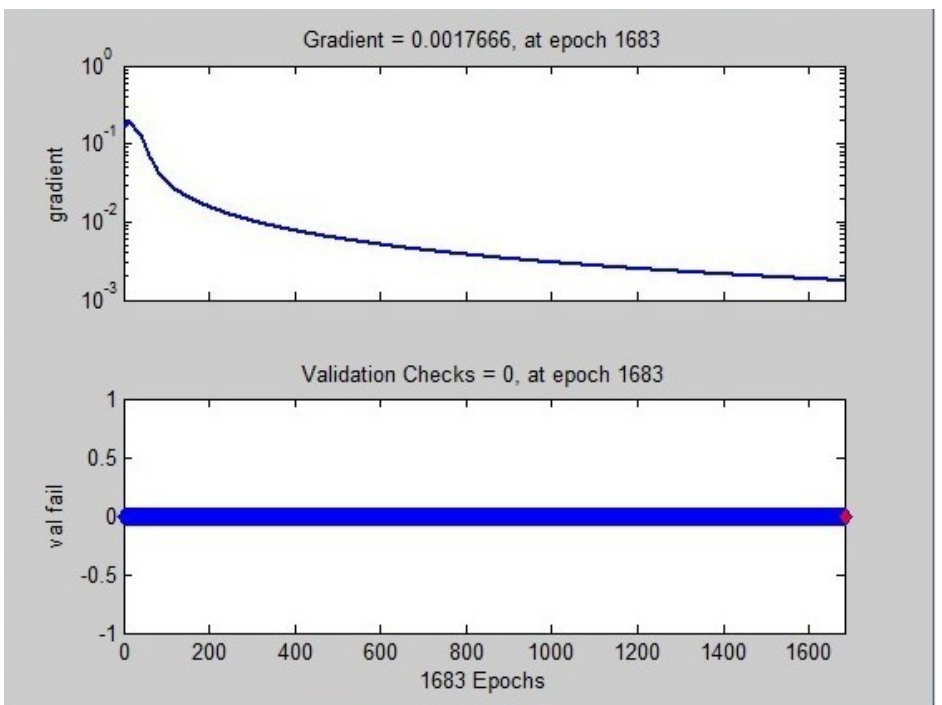

Fig.3: Training state diagram

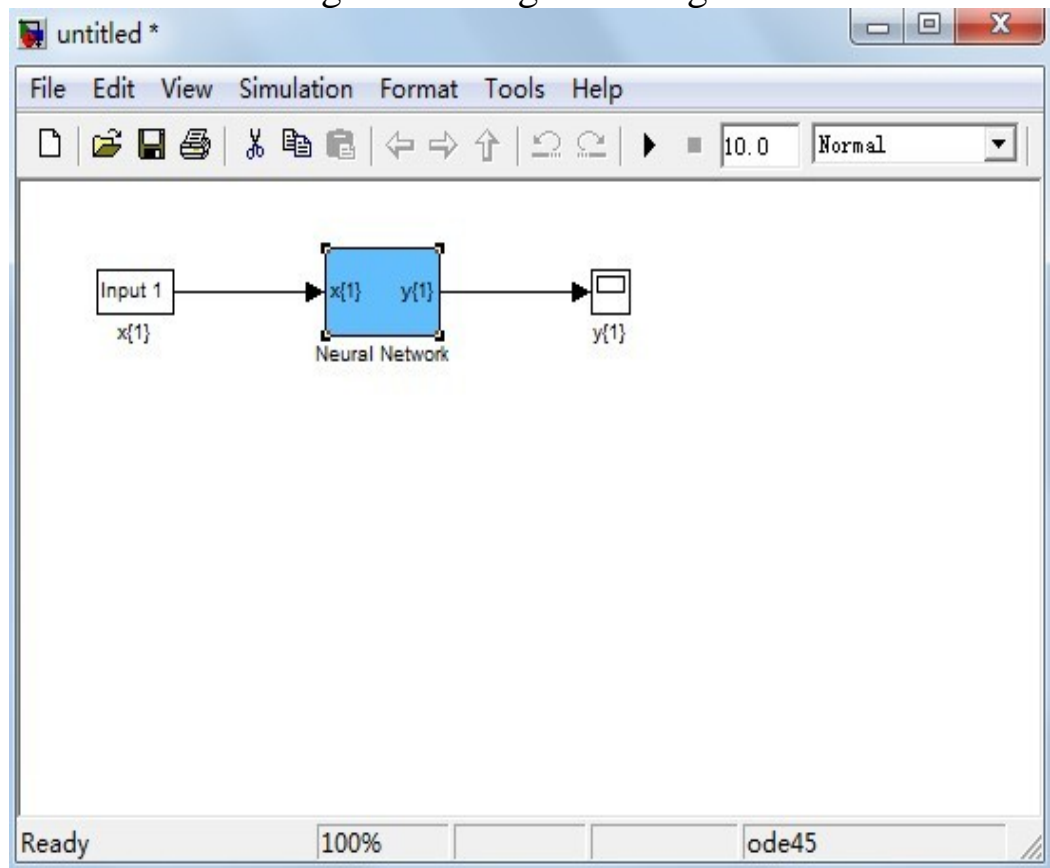

Fig.4: The system simulation

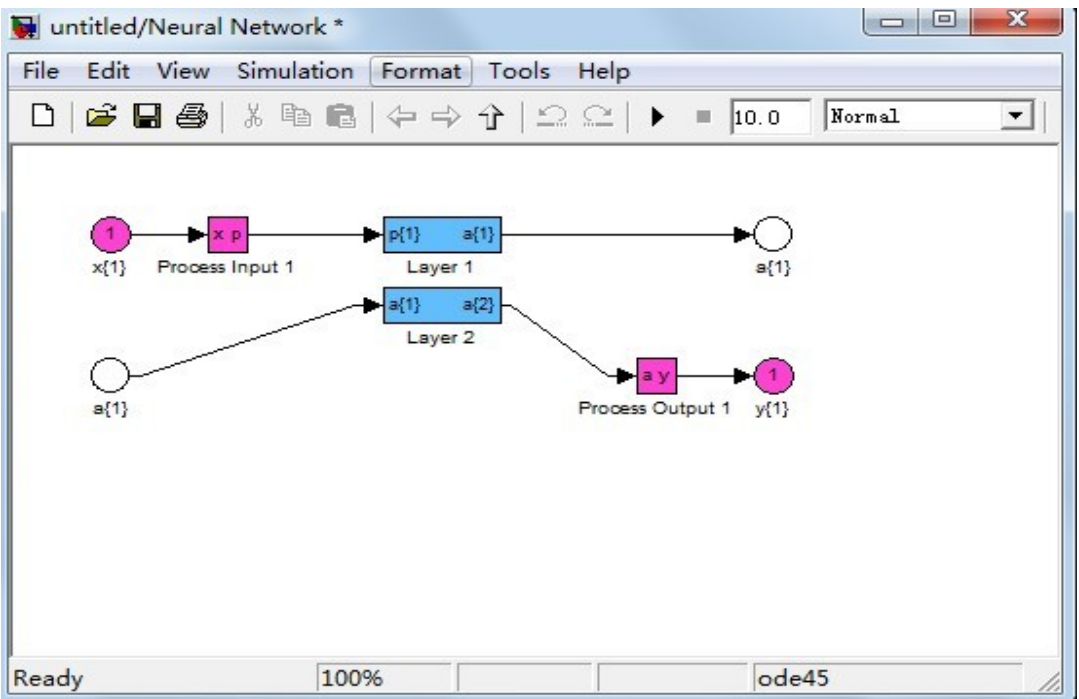

Fig.5: Neural network simulation 
Based on the MATLAB artificial intelligence code, it establishes Solidworks data interface, which can realize the feedback adjustment process of design system [10]; and uses the image design concept to design the product concept, which realizes the design process from virtual to real practical.

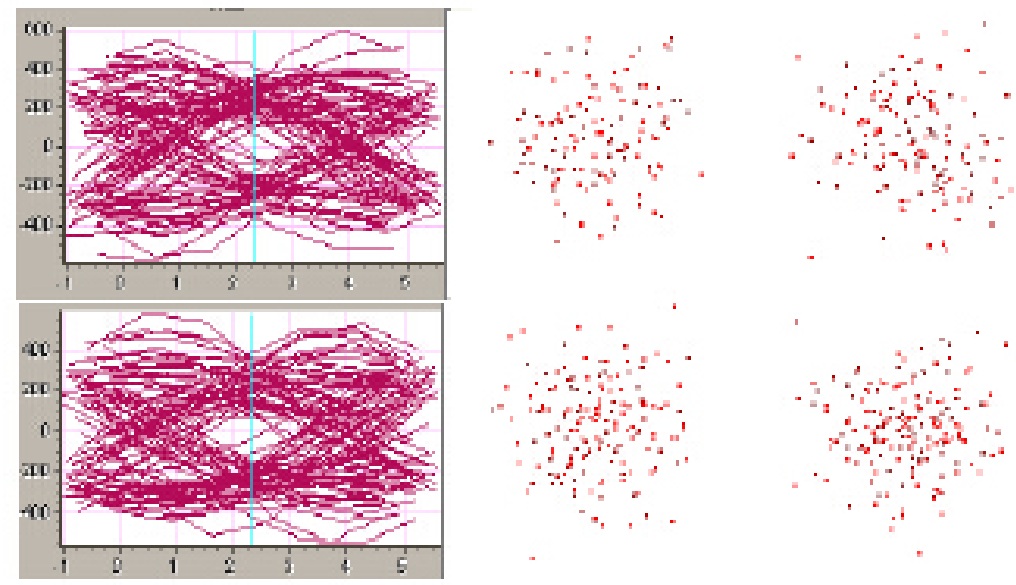

Fig.6: Cognitive image artificial intelligence process

Figure 6 shows the artificial intelligent process of cognitive image. Through the MATLAB artificial intelligent decoupling process, the processing route and optimization points of product modeling is obtained, compensating product art modeling image and adding the concept design function, finally the product image design model is obtained.

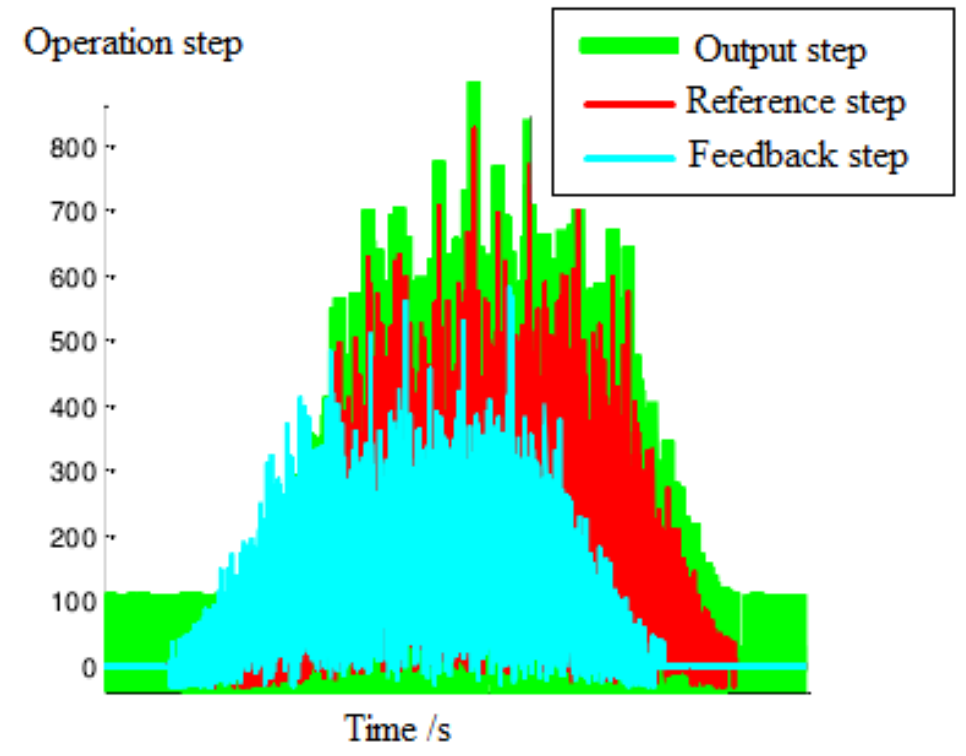

Fig.7: Artificial intelligent decoupling process of product art design

Figure 7 shows the artificial intelligent decoupling process of product art design. In art appearance image design process, reference to standard design process, it uses feedback regulation of appearance image nerve to do optimization design on the appearance. The design example of product appearance is as shown in Figure 8. 


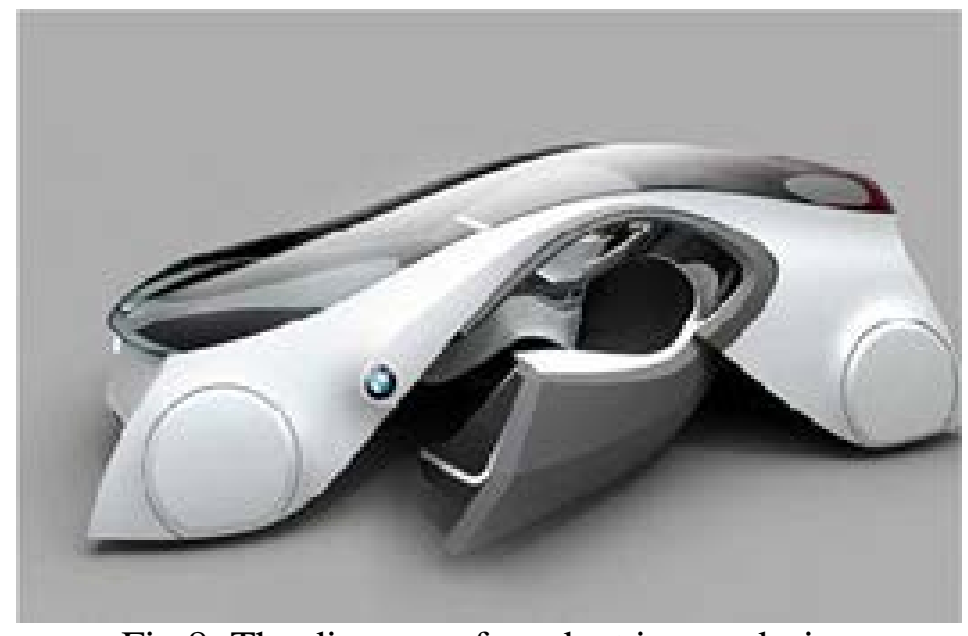

Fig.8: The diagram of product image design

Figure 8 shows the example chart of product image design [10]. It uses MATLAB software to do visualization development on the operation interface, and uses artificial intelligence structure in the feedback adjustment process, so the product appearance can be done image design, and eventually realizes the automatic design process of appearance.

\section{Summary}

This paper designs artificial intelligence virtual method of product artistic modeling, and establishes the fuzzy variable control output model. It uses MATLAB simulation software to encode the intelligent control, and designs the Solidworks data interface, which implements the artificial intelligence visual design process of product modeling. Through the analysis of the design process and design case, artificial intelligent decoupling algorithm can effectively do artificial intelligence feedback regulation on the product design process, which enhances the expression of art product image and provides a new computer method for product modeling design. But the application of this algorithm in practical product design still needs to study, through further study, so it can provide an efficient method for product modeling design.

\section{References}

[1] J. Zhu, Y.C Guo. Combining spatial diversity decision feedback blind equalization algorithm based on the maximum ratio. Journal of system simulation, 2012, 20(11): 2843-2850.

[2] Y.M. Fu, J. Zhu, Y.J. Ju. Parameters design and performance analysis of Gardner timing synchronization loop. Communication engineering, 2012, 2(6): 191-200.

[3] B.W. Luo, M.K. Wan, H. Yu. FDOA estimation algorithm based on adaptive phase compensation. Data acquisition and processing, 2012, 3(1): 20-26.

[4] C.L. Chen. Key technologies research and realization of random distributed multi antenna arrays. The PLA Information Engineering University, 203: 2-16.

[5] M.H. Hao, F. Song, S.H. Hao, B.Y. Song. NC instruction interpretation on NC simulation system system. Machine tool \& hydraulics, 2012, 3(10): 176-178.

[6] W.X. Cao, X.M. Feng. The research status and development trend of industrial robot. Machinery manufacturing, 2012, 49(2): 41-43.

[7] Y.H. Zhang, J. Zhao, L. Zhang. A novel modular reconfigurable robot system. Chinese Journal of mechanical engineering, 2012, 42(5): 175-177.

[8] L.H. Wang, H. Zhou. Research status and development direction of bionic robot. Journal of Shanghai Normal University, 2012, 36(6): 58-62.

[9] Y.J. Li, C. Ming. Situation and development strategy of our industrial robot industry. Manufacturing automation, 2012, 33(12): 106-108.

[10] S.T. Ren. Design and implementation of NC code compiler. Northwestern Polytechnical University, 2012: 2-18. 